\title{
Optimality Conditions and Duality of Three Kinds of Nonlinear Fractional Programming Problems
}

\author{
Xiaomin Zhang and Zezhong Wu \\ Department of Mathematics, Chengdu University of Information Technology, Sichuan 610225, China \\ Correspondence should be addressed to Xiaomin Zhang; zhangxiaomin228@163.com
}

Received 5 April 2013; Accepted 24 October 2013

Academic Editor: Ching-Jong Liao

Copyright (c) 2013 X. Zhang and Z. Wu. This is an open access article distributed under the Creative Commons Attribution License, which permits unrestricted use, distribution, and reproduction in any medium, provided the original work is properly cited.

\begin{abstract}
Some assumptions for the objective functions and constraint functions are given under the conditions of convex and generalized convex, which are based on the $F$-convex, $\rho$-convex, and $(F, \rho)$-convex. The sufficiency of Kuhn-Tucker optimality conditions and appropriate duality results are proved involving $(F, \rho)$-convex, $(F, \alpha, \rho, d)$-convex, and generalized $(F, \alpha, \rho, d)$-convex functions.
\end{abstract}

\section{Introduction}

Multiobjective optimization theory is a development of numerical optimization and related to many subjects, such as nonsmooth analysis, convex analysis, nonlinear analysis, and the theory of set value. It has a wide range of applications in the fields of industrial design, economics, engineering, military, management sciences, financial investment, transport, and so forth, and now it is an interdisciplinary science branch between applied mathematics and decision sciences. Convexity plays an important role in optimization theory, and it becomes an important theoretical basis and useful tool for mathematical programming and optimization theory.

Convex function theory can be traced back to the works of Holder, Jensen, and Minkowski in the beginning of this century, but the real work that caught the attention of people is the research on game theory and mathematical programming by von Neumann and Morgenstern [1], Dantzing, and Kuhn and Tucker in the forties to fifties, and people have done a lot of intensive research about convex functions from the fifties to sixties. In the middle of the sixties convex analysis was produced, and the concept of convex function is promoted in a variety of ways, and the notion of generalized convex is given.

Fractional programming has an important significance in the optimization problems; for instance, in order to measure the production or the efficiency of a system, we should minimize a ratio of functions between a given period of time and a utilized resource in engineering and economics.

Preda [2] has established the concept of $(F, \rho)$-convex based on $F$-convex [3] and $\rho$-convex [4] and obtained some results, which are the expansion of $F$-convex and $\rho$-convex. Motivated by various concepts of convexity, Liang et al. [5] have put forward a generalized convexity, which was called $(F, \alpha, \rho, d)$-convex, which extended $(F, \rho)$-convex, and Liang et al. [6], Weir and Mond [7], Weir [8], Jeyakumar and Mond [9], Egudo [10], Preda [2], and Gulati and Islam [3] obtained some corresponding optimality conditions and applied these optimality conditions to define dual problems and derived duality theorems for single objective fractional problems and multiobjective problems. Then the definition of generalized $(F, \alpha, \rho, d)$-convex is given under the condition of $(F, \alpha, \rho, d)$-convex. However, in general, fractional programming problems are nonconvex and the Kuhn-Tucker optimality conditions are only necessary. Under what conditions are the Kuhn-Tucker conditions sufficient for the optimality of problems? This question appeals to the interests of many researchers, and those are what we should probe. Based on the former conclusions, by adding conditions to objective functions and constraint functions and by changing K-T conditions [11], the optimality conditions and dual are given involving weaker convexity conditions. The main results in this paper are based on convex and generalized convex functions and the properties of sublinear functions. 
In this paper, we will discuss sufficient optimality conditions and dual problems for three kinds of nonlinear fractional programming problems, and the paper is organized as follows.

In Sections 3.1 and 3.2, we present the Kuhn-Tucker sufficient optimality conditions and dual for nonlinear fractional programming problem and multiobjective fractional programming problem based on generalized $(F, \alpha, \rho, d)$ convex. Section 3.3 contains optimality conditions and dual for multiobjective fractional programming problem under $(F, \rho)$-convex. In these sections, I present some assumptions for the objective functions and constraint functions such that the Kuhn-Tucker optimality conditions are sufficient and obtain the corresponding duality theorem.

\section{Preliminaries}

Let $E^{n}$ be the $n$-dimensional real vector space, that is, $n$ dimensional Euclidean space, where $y=\left(y_{1}, y_{2}, \ldots, y_{n}\right)^{T}$, $z=\left(z_{1}, z_{2}, \ldots, z_{n}\right)^{T} \in E^{n}$, and provides as follows, (see [12]),

$$
\begin{aligned}
& y=z \Longleftrightarrow y_{i}=z_{i}, \quad i=1,2, \ldots, n ; \\
& y>z \Longleftrightarrow y_{i}>z_{i}, \quad i=1,2, \ldots, n ; \\
& y \geqq z \Longleftrightarrow y_{i} \geqq z_{i}, \quad i=1,2, \ldots, n .
\end{aligned}
$$

Definition 1 (see [12]). Suppose that $x_{0} \in X_{0}$; that is, if $x \notin$ $X_{0}$, such that $f(x) \leqq f\left(x_{0}\right), x_{0}$ is an efficient solution of multiobjective programming problem.

Definition 2 (see [12]). Suppose that $x_{0} \in X_{0}$; that is, if $x \notin$ $X_{0}$, such that $f(x)<f\left(x_{0}\right), x_{0}$ is a weakly efficient solution of multiobjective programming problem.

Definition 3 (see [5]). Given an open set $X_{0} \subset R^{n}$, a functional $F: X_{0} \times X_{0} \times R^{n} \rightarrow R$ is called sublinear if, for any $x, x_{0} \in X_{0}$,

$$
\begin{gathered}
F\left(x, x_{0} ; a_{1}+a_{2}\right) \leqq F\left(x, x_{0} ; a_{1}\right)+F\left(x, x_{0} ; a_{2}\right), \\
\forall a_{1}, a_{2} \in R^{n}, \\
F\left(x, x_{0} ; \alpha a\right)=\alpha F\left(x, x_{0} ; a\right), \quad \forall \alpha \in R, \alpha \geqq 0, \forall a \in R^{n} .
\end{gathered}
$$

It follows from the second equality that

$$
\begin{array}{r}
F\left(x, x_{0} ; 0\right)=F\left(x, x_{0} ; 0 \times a\right)=0 \times F\left(x, x_{0} ; a\right)=0, \\
\text { for any } a \in R^{n} .
\end{array}
$$

Let $F: X_{0} \times X_{0} \times R^{n} \rightarrow R$ be a sublinear function, and let $\alpha: X_{0} \times X_{0} \rightarrow R_{+} \backslash\{0\}, \rho=\left(\rho_{1}, \rho_{2}, \ldots, \rho_{m}\right)^{T}, \rho_{i} \in R$, $d: X_{0} \times X_{0} \rightarrow R$, and the function $f=\left(f_{1}, f_{2}, \ldots, f_{m}\right):$ $X_{0} \rightarrow R^{m}$ is differentiable at $x_{0} \in X_{0}$.

Definition 4 (see [3]). Let $\phi(x)$ be a differentiable function defined on $X_{0} \subset E^{n}$. The function $\phi(x)$ is said to be $F$-convex on $X_{0}$ with respect to $F$, if $\phi(x)-\phi(y) \geqq F_{x, y}[\nabla \phi(y)]$.
Definition 5 (see $[4,12]$ ). Let $f(x)$ be a real-valued function defined on the convex set $X_{0} \subset E^{n}$, if there exists a real number $\rho \in R$, such that

$$
\begin{aligned}
& f\left(\lambda x^{1}+(1-\lambda) x^{2}\right) \leqq \lambda f\left(x^{1}\right)+(1-\lambda) f\left(x^{2}\right) \\
& -\rho \lambda(1-\lambda)\left\|x^{1}-x^{2}\right\|^{2}
\end{aligned}
$$

for any $x^{1}, x^{2} \in X_{0}$ and any $\lambda \in[0,1]$, then the function $f(x)$ is said to be $\rho$-convex on $X_{0}$.

Especially, if $\rho=0$, then we obtain the definition of convex.

If $\rho>0$ (or $\rho<0)$ in the above definition, then we have strong convex (or weak convex).

Definition 6 (see [2]). The function $f_{i}: X_{0} \rightarrow R$ is said to be $(F, \rho)$-convex at $x_{0} \in X_{0}$, if for any $x_{0} \in X_{0}, f_{i}(x)$ satisfies the following condition:

$$
f_{i}(x)-f_{i}\left(x_{0}\right) \geqq F\left(x, x_{0} ; \nabla f_{i}\left(x_{0}\right)\right)+\rho_{i} d^{2}\left(x, x_{0}\right) .
$$

Definition 7 (see [5]). The function $f_{i}$ is said to be $\left(F, \alpha, \rho_{i}, d\right)$ convex at $x_{0} \in X_{0}$, if

$$
\begin{array}{r}
f_{i}(x)-f_{i}\left(x_{0}\right) \geqq F\left(x, x_{0} ; \alpha\left(x, x_{0}\right) \nabla f_{i}\left(x_{0}\right)\right)+\rho_{i} d^{2}\left(x, x_{0}\right), \\
\forall x \in X_{0} .
\end{array}
$$

The function $f$ is said to be $(F, \alpha, \rho, d)$-convex at $x_{0}$, if each component $f_{i}$ of $f$ is $\left(F, \alpha, \rho_{i}, d\right)$-convex at $x_{0}$.

The function $f$ is said to be $(F, \alpha, \rho, d)$-convex on $X_{0}$, if it is $(F, \alpha, \rho, d)$-convex at every point in $X_{0}$.

Definition 8. The function $f_{i}$ is said to be $\left(F, \alpha, \rho_{i}, d\right)$-quasiconvex at $x_{0}$, if $f_{i}(x) \leqq f_{i}\left(x_{0}\right) \Rightarrow F\left(x, x_{0} ; \alpha\left(x, x_{0}\right) \nabla f_{i}\left(x_{0}\right)\right) \leqq$ $-\rho_{i} d^{2}\left(x, x_{0}\right)$.

The function $f$ is said to be $(F, \alpha, \rho, d)$-quasiconvex at $x_{0}$, if each component $f_{i}$ of $f$ is $\left(F, \alpha, \rho_{i}, d\right)$-quasiconvex at $x_{0}$.

Definition 9. The function $f_{i}$ is said to be $\left(F, \alpha, \rho_{i}, d\right)$ pseudoconvex at $x_{0}$, if for all $x \in X_{0}, f_{i}(x)<f_{i}\left(x_{0}\right) \Rightarrow$ $F\left(x, x_{0} ; \alpha\left(x, x_{0}\right) \nabla f_{i}\left(x_{0}\right)\right)<-\rho_{i} d^{2}\left(x, x_{0}\right)$.

The function $f$ is said to be $(F, \alpha, \rho, d)$-pseudoconvex at $x_{0}$, if each component $f_{i}$ of $f$ is $\left(F, \alpha, \rho_{i}, d\right)$-pseudoconvex at $x_{0}$.

Definition 10. The function $f$ is said to be strictly $\left(F, \alpha, \rho_{i}, d\right)$-pseudoconvex at $x_{0} \in X_{0}$, if $f(x) \leqq$ $f\left(x_{0}\right) \Rightarrow F\left(x, x_{0} ; \alpha\left(x, x_{0}\right) \nabla f\left(x_{0}\right)\right)<-\rho d^{2}\left(x, x_{0}\right)$, where $F\left(x, x_{0} ; \alpha\left(x, x_{0}\right) \nabla f\left(x_{0}\right)\right)=\left(F\left(x, x_{0} ; \alpha\left(x, x_{0}\right) \nabla f_{1}\left(x_{0}\right)\right), \ldots\right.$, $\left.F\left(x, x_{0} ; \alpha\left(x, x_{0}\right) \nabla f_{m}\left(x_{0}\right)\right)\right)$.

Further, $f$ is said to be weakly strictly $(F, \alpha, \rho, d)$ pseudoconvex at $x_{0} \in X_{0}$, if $f(x) \leqq f\left(x_{0}\right) \Rightarrow F\left(x, x_{0}\right.$; $\left.\alpha\left(x, x_{0}\right) \nabla f\left(x_{0}\right)\right)<-\rho d^{2}\left(x, x_{0}\right)$.

In order to prove our main result, we need a lemma which we present in this section. 
Lemma 11 (see [13]). Suppose that differentiable real-valued functions $h_{j}(x)(j=1,2, \ldots, m)$ are $\left(F, \alpha, \rho_{j}, d\right)$-quasiconvex at $\bar{x} \in S$; then $V^{T} h(x)$ is $\left(F, \alpha, \sum_{j=1}^{m} v_{j} \rho_{j}, d\right)$-quasiconvex at $\bar{x} \in S$, where $\rho_{j} \in R, V \geqq 0$ and $V^{T}$ denote the transpose of the $m$-dimensional column vector $V$; that is, $V^{T}=\left(v_{1}, v_{2}, \ldots, v_{m}\right)$.

\section{Optimality Conditions and Duality}

3.1. Nonlinear Fractional Programming Problem Involved Inequality and Equality Constraints Based on Generalized $(F, \alpha, \rho, d)$-Convex. Consider the nonlinear fractional programming problem (FP)

$$
\begin{array}{ll}
\min & \frac{f(x)}{g(x)} \\
\text { s.t. } & h(x) \leqq 0, l(x)=0, x \in X_{0},
\end{array}
$$

where $X_{0}$ is an open set of $R^{n}, f(x)$ and $g(x)$ are real-valued functions defined on $X_{0}, h(x)$ is an $m$-dimensional vectorvalued functions defined also on $X_{0}$, and $l(x)$ a $q$-dimensional vector-valued function.

Let

$$
S=\left\{x \in X_{0} \mid h(x) \leqq 0, l(x)=0\right\}
$$

denotes the set of all feasible solutions for (FP) and assume that $f(x), g(x), h_{j}(x)(j=1,2, \ldots, m)$, and $l_{i}(x)(i=$ $1,2, \ldots, q)$ are continuously differentiable over $X_{0}$ and that $f(x) \geqq 0, g(x)>0$, for all $x \in X_{0}$.

If $\bar{x} \in X_{0}$ is a solution for problem (FP) and if a constraint qualification [14] holds, then the Kuhn-Tucker necessary conditions are given below: there exists $V_{0} \in R^{m}$ and $W_{0} \in R^{q}$ such that

$$
\begin{gathered}
\nabla\left(\frac{f(\bar{x})}{g(\bar{x})}\right)+\nabla h(\bar{x}) V_{0}+\nabla l(\bar{x}) W_{0}=0, \\
V_{0}^{T} h(\bar{x})=0, \\
V_{0} \geqq 0, \quad h(\bar{x}) \leqq 0, \quad l(\bar{x})=0 .
\end{gathered}
$$

Theorem 12. Suppose that $\bar{x}$ is a feasible solution of $(F P)$, that the Kuhn-Tucker conditions hold at $\bar{x}$, that $f(x) / g(x)$ in problem $(F P)$ is $(F, \alpha, \rho, d)$-pseudoconvex on $S, h_{j}(x)(j=$ $1,2, \ldots, m)$ are $\left(F, \alpha, \rho_{j}, d\right)$-quasiconvex on $S$, and that $l_{i}(x)(i=1,2, \ldots, q)$ are $\left(F, \alpha, \rho_{i}, d\right)$-quasiconvex over $S, \rho, \rho_{i}$, $\rho_{j} \in R, \rho+V_{0}^{T} \rho^{\prime}+W_{0}^{T} \rho^{\prime \prime} \geqq 0$, where $V_{0}^{T} \rho^{\prime}$ is the inner product about $V_{0}$ and $\rho^{\prime}$ and $W_{0}^{T} \rho^{\prime \prime}$ the inner product about $W_{0}$ and $\rho^{\prime \prime}$. Then, $\bar{x}$ is an optimality solution for problem (FP).

Proof. Suppose that $\bar{x}$ is not an optimality solution of (FP). Then, there exists a feasible solution $x \in S$ such that $f(x) / g(x)<f(\bar{x}) / g(\bar{x})$.

By the $(F, \alpha, \rho, d)$-pseudoconvexity assumption of $f(x) / g(x)$, we have

$$
F\left(x, \bar{x} ; \alpha(x, \bar{x}) \nabla\left(\frac{f(\bar{x})}{g(\bar{x})}\right)\right)<-\rho d^{2}(x, \bar{x}) .
$$

For each $j(j=1,2, \ldots, m)$, by the $\left(F, \alpha, \rho_{j}, d\right)$ quasiconvexity assumption of $h_{j}(x)$ and Lemma 11, we have that $V_{0}^{T} h(x)$ is $\left(F, \alpha, \sum_{j=1}^{m} v_{j} \rho_{j}, d\right)$-quasiconvex on $S$. Therefore,

$$
F\left(x, \bar{x} ; \alpha(x, \bar{x}) \nabla h(\bar{x}) V_{0}\right) \leqq-\sum_{j=1}^{m} \rho_{j} v_{j} d^{2}(x, \bar{x}),
$$

that is, $F\left(x, \bar{x} ; \alpha(x, \bar{x}) \nabla h(\bar{x}) V_{0}\right) \leqq-V_{0}^{T} \rho^{\prime} d^{2}(x, \bar{x})$.

By the $\left(F, \alpha, \rho_{i}, d\right)$-quasiconvexity of $l_{i}(x)(i=1,2, \ldots, q)$ over $S$, we have that $W_{0}^{T} l(x)$ is $\left(F, \alpha, \sum_{i=1}^{q} w_{i} \rho_{i}, d\right)$ quasiconvexity on $S$.

Then we obtain $F\left(x, \bar{x} ; \alpha(x, \bar{x}) \nabla l(\bar{x}) W_{0}\right) \leqq$ $-\sum_{i=1}^{q} \rho_{i} w_{i} d^{2}(x, \bar{x})$, that is,

$$
F\left(x, \bar{x} ; \alpha(x, \bar{x}) \nabla l(\bar{x}) W_{0}\right) \leqq-W_{0}^{T} \rho^{\prime \prime} d^{2}(x, \bar{x}) .
$$

By (10), (11), and (12), and based on the sublinearity of $F$, we have

$$
\begin{gathered}
F\left(x, \bar{x} ; \alpha(x, \bar{x})\left(\nabla\left(\frac{f(\bar{x})}{g(\bar{x})}\right)+\nabla h(\bar{x}) V_{0}+\nabla l(\bar{x}) W_{0}\right)\right) \\
<-\left(\rho+V_{0}^{T} \rho^{\prime}+W_{0}^{T} \rho^{\prime \prime}\right) d^{2}(x, \bar{x}) .
\end{gathered}
$$

Considering that $\rho+V_{0}^{T} \rho^{\prime}+W_{0}^{T} \rho^{\prime \prime} \geqq 0$, we get

$$
F\left(x, \bar{x} ; \alpha(x, \bar{x})\left(\nabla\left(\frac{f(\bar{x})}{g(\bar{x})}\right)+\nabla h(\bar{x}) V_{0}+\nabla l(\bar{x}) W_{0}\right)\right)<0 .
$$

By the K-T conditions, we have $\nabla(f(\bar{x}) / g(\bar{x}))+\nabla h(\bar{x}) V_{0}+$ $\nabla l(\bar{x}) W_{0}=0$.

Hence, based on the sublinearity of $F$, we obtain

$$
F\left(x, \bar{x} ; \alpha(x, \bar{x})\left(\nabla\left(\frac{f(\bar{x})}{g(\bar{x})}\right)+\nabla h(\bar{x}) V_{0}+\nabla l(\bar{x}) W_{0}\right)\right)=0,
$$

which contradicts (14). The proof is complete.

Consider the dual problem of (FP):

$$
\begin{array}{ll}
\max & \frac{f(y)}{g(y)}+u^{T} h(y)+v^{T} l(y) \\
\text { s.t. } & \lambda^{T} \nabla\left(\frac{f(y)}{g(y)}\right)+u^{T} \nabla h(y)+v^{T} \nabla l(y)=0, \\
& u^{T} h(y) \geqq 0, \\
& v^{T} l(y)=0, \\
& u \geqq 0, \quad v \geqq 0 .
\end{array}
$$

Theorem 13. Suppose that $\lambda^{T}(f(y) / g(y))$ is $\left(F, \alpha, \rho_{1}, d\right)$ pseudoconvex at $y, u^{T} h(y)+v^{T} l(y)$ is $\left(F, \alpha, \rho_{2}, d\right)$-quasiconvex at $y$ in problem $(F P)$ and $(F D)$, and that $\rho_{1}+\rho_{2} \geqq 0$; then $\lambda^{T}(f(x) / g(x)) \geqq \lambda^{T}(f(y) / g(y))$, for any feasible solution $x$ of $(F P)$ and $(y, \lambda, u, v)$ of $(F D)$. 
Proof. Assume that the conclusion is not true; that is, $\lambda^{T}(f(x) / g(x))<\lambda^{T}(f(y) / g(y))$.

By the $\left(F, \alpha, \rho_{1}, d\right)$-pseudoconvex of $\lambda^{T}(f(x) / g(x))$ at $y$, we get

$$
F\left(x, y ; \alpha(x, y) \lambda^{T} \nabla\left(\frac{f(y)}{g(y)}\right)\right)<-\rho_{1} d^{2}(x, y) .
$$

Using $u^{T} h(x) \leqq 0, v^{T} l(x)=0, u^{T} h(y) \geqq 0, v^{T} l(y)=0$, we have

$$
u^{T} h(x)+v^{T} l(x) \leqq u^{T} h(y)+v^{T} l(y)
$$

By the $\left(F, \alpha, \rho_{2}, d\right)$-quasiconvex of $u^{T} h(y)+v^{T} l(y)$, we get

$$
F\left(x, y ; \alpha(x, y)\left(u^{T} \nabla h(y)+v^{T} \nabla l(y)\right)\right) \leqq-\rho_{2} d^{2}(x, y) .
$$

By (16) and (18) and based on the sublinearity of $F$, we have

$$
\begin{gathered}
F\left(x, y ; \alpha(x, y)\left(\lambda^{T} \nabla\left(\frac{f(y)}{g(y)}\right)\right.\right. \\
\left.\left.+u^{T} \nabla h(y)+v^{T} \nabla l(y)\right)\right) \\
<-\left(\rho_{1}+\rho_{2}\right) d^{2}(x, y) .
\end{gathered}
$$

Since $(y, \lambda, u, v)$ is the feasible solution of (FD), so

$$
\bar{\lambda}^{T} \nabla\left(\frac{f(y)}{g(y)}\right)+u^{T} \nabla h(y)+v^{T} \nabla l(y)=0 .
$$

Hence,

$$
\begin{aligned}
& 0=F(x, y ; \alpha(x, y)( \lambda^{T} \nabla\left(\frac{f(y)}{g(y)}\right) \\
&\left.\left.+u^{T} \nabla h(y)+v^{T} \nabla l(y)\right)\right) \\
&<-\left(\rho_{1}+\rho_{2}\right) d^{2}(x, y),
\end{aligned}
$$

which contradicts the known condition $\rho_{1}+\rho_{2} \geqq 0$. The proof is complete.

3.2. Nonlinear Multiobjective Fractional Programming Problem Involved Inequality and Equality Constraints Based on $(F, \alpha, \rho, d)$-Convex and Generalized $(F, \alpha, \rho, d)$-Convex. Consider the nonlinear multiobjective fractional programming problem (VFP)

$$
\begin{array}{ll}
\min & M(x)=\frac{f(x)}{g(x)}=\left[\frac{f_{1}(x)}{g(x)}, \frac{f_{2}(x)}{g(x)}, \ldots, \frac{f_{p}(x)}{g(x)}\right] \\
\text { s.t. } & h(x) \leqq 0, x \in X_{0} \\
& l(x)=0, x \in X_{0},
\end{array}
$$

where $X_{0}$ is an open set of $R^{n}, f_{i}(x)(i=1,2, \ldots, p)$, $g(x), h_{j}(x): X_{0} \rightarrow R(j=1,2, \ldots, m)$ are real-valued functions defined on $X_{0}, l_{k}(x): X_{0} \rightarrow R(k=1,2, \ldots, q)$ are real-valued functions defined also on $X_{0}, f(x)=$ $\left(f_{1}(x), f_{2}(x), \ldots, f_{p}(x)\right)^{T}, h(x)=\left(h_{1}(x), h_{2}(x), \ldots, h_{m}(x)\right)^{T}$, and $l(x)=\left(l_{1}(x), l_{2}(x), \ldots, l_{q}(x)\right)^{T}$.

Let

$$
S=\left\{x \mid x \in X_{0}, h(x) \leqq 0, l(x)=0\right\}
$$

denotes the set of all feasible solutions of (VFP) and assume that $f_{i}(x)(i=1,2, \ldots, p), g(x), h_{j}(x)(j=1,2, \ldots, m)$, and $l_{k}(x)(k=1,2, \ldots, q)$ are continuously differentiable over $X_{0}$ and that $g(x)>0$, for all $x \in X_{0}$.

Theorem 14. Suppose that $f(x) / g(x)$ is weakly strictly $\left(F, \alpha, \rho_{1}, d\right)$-pseudoconvex at $\bar{x} \in X_{0}, h_{j}(x)(j=1,2, \ldots, m)$ are $\left(F, \alpha, \rho_{2}, d\right)$-quasiconvex with respect to $\bar{x} \in X_{0}, l_{k}(x)$ ( $k=$ $1,2, \ldots, q)$ are $\left(F, \alpha, \rho_{3}, d\right)$-convex with respect to $\bar{x} \in X_{0}$, and that there exists $\bar{\lambda} \in \wedge^{++}\left(\right.$or $\left.\wedge^{+}\right), \bar{u} \in R_{+}^{m}, \bar{v} \in R_{+}^{q}$ satisfying

$$
\begin{aligned}
& \sum_{i=1}^{p} \bar{\lambda}_{i} \nabla\left(\frac{f(\bar{x})}{g(\bar{x})}\right)+ \sum_{j=1}^{m} \bar{u}_{j} \nabla h_{j}(\bar{x})+\sum_{k=1}^{q} \bar{v}_{k} \nabla l_{k}(\bar{x})=0, \\
& \bar{u}_{j} h_{j}(\bar{x})=0, \\
& h_{j}(x) \leqq 0, \quad j=1,2, \ldots, m, \\
& l_{k}(x)=0, \quad k=1,2, \ldots, q
\end{aligned}
$$

and $\rho=\sum_{i=1}^{p} \bar{\lambda}_{i} \rho_{1}+\sum_{j=1}^{m} \bar{u}_{j} \rho_{2}+\sum_{k=1}^{q} \bar{v}_{k} \rho_{3} \geqq 0$. Then, $\bar{x}$ is an efficient solution of (VFP), where

$\wedge^{+}$

$$
\begin{aligned}
& =\left\{\bar{\lambda}=\left(\bar{\lambda}_{1}, \bar{\lambda}_{2}, \ldots, \bar{\lambda}_{p}\right)^{T} \mid \bar{\lambda}_{i} \geqq 0, i=1,2, \ldots, p \cdot \sum_{i=1}^{p} \bar{\lambda}_{i}=1\right\}, \\
& \wedge^{++} \\
& =\left\{\bar{\lambda}=\left(\bar{\lambda}_{1}, \bar{\lambda}_{2}, \ldots, \bar{\lambda}_{p}\right)^{T} \mid \bar{\lambda}_{i}>0, i=1,2, \ldots, p \cdot \sum_{i=1}^{p} \bar{\lambda}_{i}=1\right\} .
\end{aligned}
$$

Proof. Suppose that $\bar{x}$ is not an efficient solution of (VFP); then there exists a feasible solution $x \in X_{0}$ such that $M(x) \leqq$ $M(\bar{x})$, that is, $f(x) / g(x) \leqq f(\bar{x}) / g(\bar{x})$.

By the weakly strict $\left(F, \alpha, \rho_{1}, d\right)$-pseudoconvexity of $f(x) / g(x)$ at $\bar{x} \in X_{0}$, we get

$$
F\left(x, \bar{x} ; \alpha(x, \bar{x}) \nabla\left(\frac{f(\bar{x})}{g(\bar{x})}\right)\right)<-\rho_{1} d^{2}(x, \bar{x}) .
$$

Using $\bar{\lambda} \in \wedge^{++}\left(\right.$or $\left.\wedge^{+}\right)$, then we have

$$
\sum_{i=1}^{p} \bar{\lambda}_{i} F\left(x, \bar{x} ; \alpha(x, \bar{x}) \nabla\left(\frac{f(\bar{x})}{g(\bar{x})}\right)\right)<-\sum_{i=1}^{p} \bar{\lambda}_{i} \rho_{1} d^{2}(x, \bar{x}) .
$$


Based on the sublinearity of $F$, we obtain

$$
F\left(x, \bar{x} ; \alpha(x, \bar{x}) \sum_{i=1}^{p} \bar{\lambda}_{i} \nabla\left(\frac{f(\bar{x})}{g(\bar{x})}\right)\right)<-\sum_{i=1}^{p} \bar{\lambda}_{i} \rho_{1} d^{2}(x, \bar{x}) .
$$

Since $\bar{u}^{T} h(\bar{x})=0, \bar{u} \geqq 0$ and $h_{j}(x) \leqq 0$, we have $\bar{u}^{T} h(x)-$ $\bar{u}^{T} h(\bar{x}) \leqq 0$; that is, $\bar{u}^{T} h(x) \leqq \bar{u}^{T} h(\bar{x})$.

Since $h_{j}(x)(j=1,2, \ldots, m)$ are $\left(F, \alpha, \rho_{2}, d\right)$-quasiconvex at $\bar{x} \in X_{0}$, by Lemma 11 , we have $\bar{u}^{T} h$ is $\left(F, \alpha, \rho_{2} \sum_{j=1}^{m} \bar{u}_{j}, d\right)$ quasiconvex at $\bar{x} \in X_{0}$.

Hence, we obtain the following inequality:

$$
F\left(x, \bar{x} ; \alpha(x, \bar{x}) \sum_{j=1}^{m} \bar{u}_{j} \nabla h_{j}(\bar{x})\right) \leqq-\sum_{j=1}^{m} \bar{u}_{j} \rho_{2} d^{2}(x, \bar{x}) .
$$

By the $\left(F, \alpha, \rho_{3}, d\right)$-convexity of $l_{k}(x)$ at $\bar{x} \in X_{0}$, we have

$$
l_{k}(x)-l_{k}(\bar{x}) \geqq F\left(x, \bar{x} ; \alpha(x, \bar{x}) \nabla l_{k}(\bar{x})\right)+\rho_{3} d^{2}(x, \bar{x}) .
$$

Since $\bar{v} \geqq 0$, we have

$$
\begin{aligned}
0=\bar{v} l(x)-\bar{v} l(\bar{x}) \geqq & \sum_{k=1}^{q} \bar{v}_{k} F\left(x, \bar{x} ; \alpha(x, \bar{x}) \nabla l_{k}(\bar{x})\right) \\
& +\sum_{k=1}^{q} \bar{v}_{k} \rho_{3} d^{2}(x, \bar{x}) .
\end{aligned}
$$

By the sublinearity of $F$, we obtain

$$
F\left(x, \bar{x} ; \alpha(x, \bar{x}) \sum_{k=1}^{q} \bar{v}_{k} \nabla l_{k}(\bar{x})\right) \leqq-\sum_{k=1}^{q} \bar{v}_{k} \rho_{3} d^{2}(x, \bar{x}) .
$$

By the known conditions, we have

$$
\begin{gathered}
F\left(x, \bar{x} ; \alpha(x, \bar{x})\left(\sum_{i=1}^{p} \bar{\lambda}_{i} \nabla\left(\frac{f(\bar{x})}{g(\bar{x})}\right)+\sum_{j=1}^{m} \bar{u}_{j} \nabla h_{j}(\bar{x})\right.\right. \\
\left.\left.+\sum_{k=1}^{q} \bar{v}_{k} \nabla l_{k}(\bar{x})\right)\right)=0 .
\end{gathered}
$$

By (28), (29), and (32) and by the sublinearity of $F$, we obtain

$$
\begin{gathered}
F\left(x, \bar{x} ; \alpha(x, \bar{x})\left(\sum_{i=1}^{p} \bar{\lambda}_{i} \nabla\left(\frac{f(\bar{x})}{g(\bar{x})}\right)+\sum_{j=1}^{m} \bar{u}_{j} \nabla h_{j}(\bar{x})\right.\right. \\
\left.\left.+\sum_{k=1}^{q} \bar{v}_{k} \nabla l_{k}(\bar{x})\right)\right) \\
<-\left(\sum_{i=1}^{p} \bar{\lambda}_{i} \rho_{1}+\sum_{j=1}^{m} \bar{u}_{j} \rho_{2}+\sum_{k=1}^{q} \bar{v}_{k} \rho_{3}\right) d^{2}(x, \bar{x}) \\
=-\rho d^{2}(x, \bar{x}) \leqq 0
\end{gathered}
$$

which contradicts the fact of (33). Therefore, $\bar{x}$ is an efficient solution of (VFP). The proof is complete.
Consider the dual problem of (VFP)

$$
\begin{array}{ll}
\max \quad\left(\frac{f_{1}(y)}{g(y)}+u^{T} h(y)+v^{T} l(y), \ldots,\right. \\
& \left.\frac{f_{p}(y)}{g(y)}+u^{T} h(y)+v^{T} l(y)\right) \\
\text { s.t. } \quad & \sum_{i=1}^{p} \lambda_{i} \nabla\left(\frac{f_{i}(y)}{g(y)}\right)+\sum_{j=1}^{m} u_{j} \nabla h_{j}(y) \\
& +\sum_{k=1}^{q} v_{k} \nabla l_{k}(y)=0 \\
& \lambda \in \Lambda^{++}, \quad u \in R_{+}^{m}, \quad v \in R_{+}^{q} .
\end{array}
$$

Theorem 15. $\left(f_{i} / g\right)(i=1,2, \ldots, p)$ is $\left(F, \alpha, \rho_{1}, d\right)$-convex at $y, h_{j}(j=1,2, \ldots, m)$ is $\left(F, \alpha, \rho_{2}, d\right)$-convex at $y$, $l_{k}(k=1,2, \ldots, q)$ is $\left(F, \alpha, \rho_{3}, d\right)$-convex at $y$, and $\sum_{i=1}^{p} \lambda_{i} \rho_{1}+$ $\sum_{j=1}^{m} u_{j} \rho_{2}+\sum_{k=1}^{q} v_{k} \rho_{3} \geqq 0$, then

$$
\sum_{i=1}^{p} \lambda_{i}\left(\frac{f_{i}(x)}{g(x)}\right) \geqq \sum_{i=1}^{p} \lambda_{i}\left(\frac{f_{i}(y)}{g(y)}\right)+u^{T} h(y)+v^{T} l(y) .
$$

Proof. By the $\left(F, \alpha, \rho_{1}, d\right)$-convex of $f_{i} / g$ at $y$, we get

$$
\begin{aligned}
\frac{f_{i}(x)}{g(x)}-\frac{f_{i}(y)}{g(y)} \geqq & F\left(x, y ; \alpha(x, y) \nabla\left(\frac{f_{i}(y)}{g(y)}\right)\right) \\
& +\rho_{1} d^{2}(x, y), \quad i=1,2, \ldots, p .
\end{aligned}
$$

By the $\left(F, \alpha, \rho_{2}, d\right)$-convex of $h_{j}$ at $y$, we get

$$
\begin{array}{r}
h_{j}(x)-h_{j}(y) \geqq F\left(x, y ; \alpha(x, y) \nabla h_{j}(y)\right)+\rho_{2} d^{2}(x, y), \\
j=1,2, \ldots, m .
\end{array}
$$

By the $\left(F, \alpha, \rho_{3}, d\right)$-convex of $l_{k}$ at $y$, we get

$$
\begin{array}{r}
l_{k}(x)-l_{k}(y) \geqq F\left(x, y ; \alpha(x, y) \nabla l_{k}(y)\right)+\rho_{3} d^{2}(x, y), \\
k=1,2, \ldots, q .
\end{array}
$$


Since $\lambda \in \Lambda^{++}, u \in R_{+}^{m}, v \in R_{+}^{q}$, and by the previous three inequalities, we have that

$$
\begin{aligned}
& \left(\sum_{i=1}^{p} \lambda_{i}\left(\frac{f_{i}(x)}{g(x)}\right)+u^{T} h(x)+v^{T} l(x)\right) \\
& -\left(\sum_{i=1}^{p} \lambda_{i}\left(\frac{f_{i}(y)}{g(y)}\right)+u^{T} h(y)+v^{T} l(y)\right) \\
& \geqq \sum_{i=1}^{p} \lambda_{i} F\left(x, y ; \alpha(x, y) \nabla\left(\frac{f_{i}(y)}{g(y)}\right)\right) \\
& \quad+\sum_{j=1}^{m} u_{j} F\left(x, y ; \alpha(x, y) \nabla h_{j}(y)\right) \\
& \quad+\sum_{k=1}^{q} v_{k} F\left(x, y ; \alpha(x, y) \nabla l_{k}(y)\right) \\
& \quad+\left(\sum_{i=1}^{p} \lambda_{i} \rho_{1}+\sum_{j=1}^{m} u_{j} \rho_{2}+\sum_{k=1}^{q} v_{k} \rho_{3}\right) d^{2}(x, y) .
\end{aligned}
$$

By the sublinearity of $F$, we obtain

$$
\begin{aligned}
& \left(\sum_{i=1}^{p} \lambda_{i}\left(\frac{f_{i}(x)}{g(x)}\right)+u^{T} h(x)+v^{T} l(x)\right) \\
& -\left(\sum_{i=1}^{p} \lambda_{i}\left(\frac{f_{i}(y)}{g(y)}\right)+u^{T} h(y)+v^{T} l(y)\right) \\
& \geqq F\left(x, y ; \alpha(x, y)\left(\sum_{i=1}^{p} \lambda_{i} \nabla\left(\frac{f_{i}(y)}{g(y)}\right)+\sum_{j=1}^{m} u_{j} \nabla h_{j}(y)\right.\right. \\
& \left.\left.+\sum_{k=1}^{q} v_{k} \nabla l_{k}(y)\right)\right) \\
& +\left(\sum_{i=1}^{p} \lambda_{i} \rho_{1}+\sum_{j=1}^{m} u_{j} \rho_{2}+\sum_{k=1}^{q} v_{k} \rho_{3}\right) d^{2}(x, y) .
\end{aligned}
$$

By the feasibility of $(y, \lambda, u, v)$, we have

$$
\sum_{i=1}^{p} \lambda_{i} \nabla\left(\frac{f_{i}(y)}{g(y)}\right)+\sum_{j=1}^{m} u_{j} \nabla h_{j}(y)+\sum_{k=1}^{q} v_{k} \nabla l_{k}(y)=0 .
$$

Since $\sum_{i=1}^{p} \lambda_{i} \rho_{1}+\sum_{j=1}^{m} u_{j} \rho_{2}+\sum_{k=1}^{q} v_{k} \rho_{3} \geqq 0$ and by (41), we get

$$
\begin{aligned}
& \left(\sum_{i=1}^{p} \lambda_{i}\left(\frac{f_{i}(x)}{g(x)}\right)+u^{T} h(x)+v^{T} l(x)\right) \\
& -\left(\sum_{i=1}^{p} \lambda_{i}\left(\frac{f_{i}(y)}{g(y)}\right)+u^{T} h(y)+v^{T} l(y)\right) \geqq 0 .
\end{aligned}
$$

Since $u^{T} h(x) \leqq 0, v^{T} l(x)=0$, we obtain

$$
\sum_{i=1}^{p} \lambda_{i}\left(\frac{f_{i}(x)}{g(x)}\right) \geqq \sum_{i=1}^{p} \lambda_{i}\left(\frac{f_{i}(y)}{g(y)}\right)+u^{T} h(y)+v^{T} l(y) .
$$

The proof is complete.

3.3. Nonlinear Multiobjective Fractional Programming Problem Involved Inequality and Equality Constraints under $(F, \rho)$ Convex. Consider the multiobjective fractional programming problem (MFP)

$$
\begin{array}{ll}
\min & \left(\frac{f_{1}(x)}{g_{1}(x)}, \frac{f_{2}(x)}{g_{2}(x)}, \ldots, \frac{f_{p}(x)}{g_{p}(x)}\right) \\
\text { s.t. } & h_{j}(x) \leqq 0, j=1,2, \ldots, m, x \in X_{0}, \\
& l_{k}(x)=0, k=1,2, \ldots, q, x \in X_{0},
\end{array}
$$

where $X_{0}$ is an open set of $R^{n}, f_{i}(x)(i=1,2, \ldots, p): X_{0} \rightarrow$ $R, f_{i}(x) \geqq 0, g_{i}(x)(i=1,2, \ldots, p): X_{0} \rightarrow R, g_{i}(x)>0$, and $h_{j}(x)(j=1,2, \ldots, m): X_{0} \rightarrow R, l_{k}(x)(k=1,2, \ldots, q):$ $X_{0} \rightarrow R$ are continuously differentiable over $X_{0}$.

Denote by $G$ the set of all feasible solutions for (MFP); that is,

$$
\begin{gathered}
G=\left\{x \in X_{0} \mid h_{j}(x) \leqq 0, j=1,2, \ldots, m ;\right. \\
\left.l_{k}(x)=0, k=1,2, \ldots, q\right\}
\end{gathered}
$$

and let $\varphi_{i}(x)=f_{i}(x) / g_{i}(x), \varphi(x)=\left(\varphi_{1}(x), \varphi_{2}(x), \ldots, \varphi_{p}(x)\right)$.

Theorem 16. Assume that there exists $(\bar{x}, \bar{\alpha}, \bar{\lambda}, \bar{v})$ and $\bar{\alpha}=$ $\left(\bar{\alpha}_{1}, \bar{\alpha}_{2}, \ldots, \bar{\alpha}_{p}\right) \in R_{+}^{p}, \bar{\lambda}=\left(\bar{\lambda}_{1}, \bar{\lambda}_{2}, \ldots, \bar{\lambda}_{m}\right), \bar{v}=\left(\bar{v}_{1}, \bar{v}_{2}, \ldots, \bar{v}_{q}\right)$ such that

(i) $\sum_{i=1}^{p} \bar{\alpha}_{i} T_{i}(\bar{x})+\sum_{j=1}^{m} \bar{\lambda}_{j} \nabla h_{j}(\bar{x})+\sum_{k=1}^{q} \bar{v}_{k} \nabla l_{k}(\bar{x})=0$,

$$
\begin{aligned}
& \bar{\lambda}_{j} h_{j}(\bar{x})=0, j=1,2, \ldots, m, \\
& h_{j}(\bar{x}) \leqq 0, j=1,2, \ldots, m, l_{k}(\bar{x})=0, k=1,2, \ldots, q, \\
& \text { where } T_{i}(\bar{x})=\left(1 / g_{i}(\bar{x})\right)\left[\nabla f_{i}(\bar{x})-\varphi_{i}(\bar{x}) \nabla g_{i}(\bar{x})\right] ;
\end{aligned}
$$

(ii) $f_{i}$ and $-g_{i}(i=1,2, \ldots, p)$ are $(F, \rho)$-convex at $\bar{x}$, and $\rho>0$;

(iii) $h_{j}$ are $(F, \rho)$-convex at $\bar{x}$ for all $j, j=1,2, \ldots, m$, and $\rho>0$;

(iv) $l_{k}$ are $(F, \rho)$-convex at $\bar{x}$ for all $k, k=1,2, \ldots, q$, and $\rho>0$.

Then $\bar{x}$ is a Pareto optimality solution of (MFP).

Proof. Suppose that $\bar{x}$ is not a Pareto optimality solution of (MFP); then there exists a feasible solution $x \in G$ such that $f_{i}(x) / g_{i}(x) \leqq f_{i}(\bar{x}) / g_{i}(\bar{x}), i=1,2, \ldots, p$, that is, $f_{i}(x)-$ $\left(f_{i}(\bar{x}) g_{i}(x)\right) / g_{i}(\bar{x}) \leqq 0$, that is, $f_{i}(x)-\left(f_{i}(\bar{x}) / g_{i}(\bar{x})\right) g_{i}(x) \leqq$ $f_{i}(\bar{x})-\left(f_{i}(\bar{x}) / g_{i}(\bar{x})\right) g_{i}(\bar{x})$; it follows that

$$
f_{i}(x)-\varphi_{i}(\bar{x}) g_{i}(x) \leqq f_{i}(\bar{x})-\varphi_{i}(\bar{x}) g_{i}(\bar{x}) .
$$


By the $(F, \rho)$-convexity of $f_{i}$ and $-g_{i}, i=1,2, \ldots, p$, we have

$$
\begin{array}{rc}
f_{i}(x)-f_{i}(\bar{x}) \geqq F\left(x, \bar{x} ; \nabla f_{i}(\bar{x})\right)+\rho d^{2}(x, \bar{x}), \quad \forall x \in X_{0}, \\
-g_{i}(x)+g_{i}(\bar{x}) \geqq F\left(x, \bar{x} ;-\nabla g_{i}(\bar{x})\right)+\rho d^{2}(x, \bar{x}), \quad \forall x \in X_{0} .
\end{array}
$$

Using the conditions $f_{i}(\bar{x}) \geqq 0, g_{i}(\bar{x})>0$, we see that $\varphi_{i}(\bar{x})=$ $f_{i}(\bar{x}) / g_{i}(\bar{x}) \geqq 0$.

By the properties of the sublinear functional $F$, we obtain

$$
\begin{aligned}
& -\varphi_{i}(\bar{x}) g_{i}(x)+\varphi_{i}(\bar{x}) g_{i}(\bar{x}) \\
& \quad \geqq \varphi_{i}(\bar{x}) F\left(x, \bar{x} ;-\nabla g_{i}(\bar{x})\right)+\varphi_{i}(\bar{x}) \rho d^{2}(x, \bar{x}) \\
& \quad=F\left(x, \bar{x} ;-\varphi_{i}(\bar{x}) \nabla g_{i}(\bar{x})\right)+\varphi_{i}(\bar{x}) \rho d^{2}(x, \bar{x}) .
\end{aligned}
$$

By (48) and (49) and based on the sublinearity of $F$, we have

$$
\begin{aligned}
f_{i}(x)-\varphi_{i}(\bar{x}) & g_{i}(x)-\left[f_{i}(\bar{x})-\varphi_{i}(\bar{x}) g_{i}(\bar{x})\right] \\
\geqq & F\left(x, \bar{x} ; \nabla f_{i}(\bar{x})-\varphi_{i}(\bar{x}) \nabla g_{i}(\bar{x})\right) \\
+ & {\left[1+\varphi_{i}(\bar{x})\right] \rho d^{2}(x, \bar{x}) . }
\end{aligned}
$$

By (47), we have

$F\left(x, \bar{x} ; \nabla f_{i}(\bar{x})-\varphi_{i}(\bar{x}) \nabla g_{i}(\bar{x})\right)+\left[1+\varphi_{i}(\bar{x})\right] \rho d^{2}(x, \bar{x}) \leqq 0$.

If we sum up after multiplying by $\bar{\alpha}_{i}\left(1 / g_{i}(\bar{x})\right) \geqq 0(i=$ $1,2, \ldots, p)$ in the above inequality and by using the sublinearity of $F$, we have

$$
\begin{gathered}
F\left(x, \bar{x} ; \sum_{i=1}^{p} \bar{\alpha}_{i}\left(\frac{1}{g_{i}(\bar{x})}\right)\left[\nabla f_{i}(\bar{x})-\varphi_{i}(\bar{x}) \nabla g_{i}(\bar{x})\right]\right) \\
+\sum_{i=1}^{p} \bar{\alpha}_{i}\left(\frac{1}{g_{i}(\bar{x})}\right)\left[1+\varphi_{i}(\bar{x})\right] \rho d^{2}(x, \bar{x}) \leqq 0 .
\end{gathered}
$$

Since $T_{i}(\bar{x})=\left(1 / g_{i}(\bar{x})\right)\left[\nabla f_{i}(\bar{x})-\varphi_{i}(\bar{x}) \nabla g_{i}(\bar{x})\right]$, we get

$$
\begin{aligned}
& F\left(x, \bar{x} ; \sum_{i=1}^{p} \bar{\alpha}_{i} T_{i}(\bar{x})\right) \\
& \quad+\sum_{i=1}^{p} \bar{\alpha}_{i}\left(\frac{1}{g_{i}(\bar{x})}\right)\left[1+\varphi_{i}(\bar{x})\right] \rho d^{2}(x, \bar{x}) \leqq 0 .
\end{aligned}
$$

On the other hand, for $j=1,2, \ldots, m$, by the $(F, \rho)$-convexity of $h_{j}$ at $\bar{x}$, we have

$$
h_{j}(x)-h_{j}(\bar{x}) \geqq F\left(x, \bar{x} ; \nabla h_{j}(\bar{x})\right)+\rho d^{2}(x, \bar{x}), \quad \forall x \in X_{0} .
$$

On multiplying the inequality (54) by $\bar{\lambda}_{j} \geq 0$ and using the sublinearity of $F$, we have

$$
\bar{\lambda}_{j} h_{j}(x)-\bar{\lambda}_{j} h_{j}(\bar{x}) \geqq F\left(x, \bar{x} ; \bar{\lambda}_{j} \nabla h_{j}(\bar{x})\right)+\bar{\lambda}_{j} \rho d^{2}(x, \bar{x}),
$$

which together with $\bar{\lambda}_{j} h_{j}(x) \leqq 0$ and $\bar{\lambda}_{j} h_{j}(\bar{x})=0$ yields

$$
F\left(x, \bar{x} ; \bar{\lambda}_{j} \nabla h_{j}(\bar{x})\right)+\bar{\lambda}_{j} \rho d^{2}(x, \bar{x}) \leqq 0 .
$$

By accumulating the inequality (56) with $j$, we have

$$
\sum_{j=1}^{m} F\left(x, \bar{x} ; \bar{\lambda}_{j} \nabla h_{j}(\bar{x})\right)+\sum_{j=1}^{m} \bar{\lambda}_{j} \rho d^{2}(x, \bar{x}) \leqq 0,
$$

that is,

$$
F\left(x, \bar{x} ; \sum_{j=1}^{m} \bar{\lambda}_{j} \nabla h_{j}(\bar{x})\right)+\sum_{j=1}^{m} \bar{\lambda}_{j} \rho d^{2}(x, \bar{x}) \leqq 0 .
$$

For $k=1,2, \ldots, q$, by the $(F, \rho)$-convexity of $l_{k}$ at $\bar{x}$, we have that

$$
l_{k}(x)-l_{k}(\bar{x}) \geqq F\left(x, \bar{x} ; \nabla l_{k}(\bar{x})\right)+\rho d^{2}(x, \bar{x}), \quad \forall x \in X_{0} .
$$

On multiplying the inequality (59) with $\bar{v}_{k} \geqq 0$, we get

$$
\bar{v}_{k} l_{k}(x)-\bar{v}_{k} l_{k}(\bar{x}) \geqq F\left(x, \bar{x} ; \bar{v}_{k} \nabla l_{k}(\bar{x})\right)+\bar{v}_{k} \rho d^{2}(x, \bar{x})
$$

which together with $\bar{v}_{k} l_{k}(x)=0$ and $\bar{v}_{k} l_{k}(\bar{x})=0$ yields

$$
F\left(x, \bar{x} ; \bar{v}_{k} \nabla l_{k}(\bar{x})\right)+\bar{v}_{k} \rho d^{2}(x, \bar{x}) \leqq 0 .
$$

By accumulating the inequality (61) with $k$, we have

$$
\sum_{k=1}^{q} F\left(x, \bar{x} ; \bar{v}_{k} \nabla l_{k}(\bar{x})\right)+\sum_{k=1}^{q} \bar{v}_{k} \rho d^{2}(x, \bar{x}) \leqq 0 .
$$

The inequality (62) along with the sublinearity of $F$ implies

$$
F\left(x, \bar{x} ; \sum_{k=1}^{q} \bar{v}_{k} \nabla l_{k}(\bar{x})\right)+\sum_{k=1}^{q} \bar{v}_{k} \rho d^{2}(x, \bar{x}) \leqq 0 .
$$

The sublinearity of $F$, (53), (58), and (63) yields

$$
\begin{gathered}
F\left(x, \bar{x} ; \sum_{i=1}^{p} \bar{\alpha}_{i} T_{i}(\bar{x})+\sum_{j=1}^{m} \bar{\lambda}_{j} \nabla h_{j}(\bar{x})+\sum_{k=1}^{q} \bar{v}_{k} \nabla l_{k}(\bar{x})\right) \\
+\sum_{i=1}^{p} \bar{\alpha}_{i}\left(\frac{1}{g_{i}(\bar{x})}\right)\left[1+\varphi_{i}(\bar{x})\right] \rho d^{2}(x, \bar{x}) \\
\quad+\sum_{j=1}^{m} \bar{\lambda}_{j} \rho d^{2}(x, \bar{x})+\sum_{k=1}^{q} \bar{v}_{k} \rho d^{2}(x, \bar{x}) \leqq 0 .
\end{gathered}
$$

According to the assumption and the sublinearity of $F$, we obtain

$$
\begin{aligned}
& F\left(x, \bar{x} ; \sum_{i=1}^{p} \bar{\alpha}_{i} T_{i}(\bar{x})+\sum_{j=1}^{m} \bar{\lambda}_{j} \nabla h_{j}(\bar{x})+\sum_{k=1}^{q} \bar{v}_{k} \nabla l_{k}(\bar{x})\right) \\
& +\sum_{i=1}^{p} \bar{\alpha}_{i}\left(\frac{1}{g_{i}(\bar{x})}\right)\left[1+\varphi_{i}(\bar{x})\right] \rho d^{2}(x, \bar{x})+\sum_{j=1}^{m} \bar{\lambda}_{j} \rho d^{2}(x, \bar{x}) \\
& +\sum_{k=1}^{q} \bar{v}_{k} \rho d^{2}(x, \bar{x})>0,
\end{aligned}
$$

which contradicts (64) obviously. 
Therefore, $\bar{x}$ is a Pareto optimality solution of (MFP). The proof is complete.

Consider the dual problem of (MFP)

$$
\begin{array}{ll}
\max \quad & \left(\frac{f_{1}(y)}{g_{1}(y)}+u^{T} h(y)+v^{T} l(y), \ldots,\right. \\
& \left.\frac{f_{p}(y)}{g_{p}(y)}+u^{T} h(y)+v^{T} l(y)\right) \\
\text { s.t. } \quad & \sum_{i=1}^{p} \lambda_{i} \nabla\left(\frac{f_{i}(y)}{g_{i}(y)}\right)+\sum_{j=1}^{m} u_{j} \nabla h_{j}(y) \\
& +\sum_{k=1}^{q} v_{k} \nabla l_{k}(y)=0, \\
& u^{T} h(y) \geqq 0, \\
& v^{T} l(y)=0, \\
& u \geqq 0, \quad v \geqq 0 .
\end{array}
$$

Theorem 17. $f_{i}(y) / g_{i}(y)(i=1,2, \ldots, p)$ is $(F, \rho)$-convex at $y, h_{j}(y)(j=1,2, \ldots, m)$ is $(F, \rho)$-convex at $y, l_{k}(y)(k=$ $1,2, \ldots, q)$ is $(F, \rho)$-convex at $y$, and $\lambda \in \Lambda^{++}, u \in R_{+}^{m}, v \in R_{+}^{q}$, $\sum_{i=1}^{p} \lambda_{i} \rho_{i}+\sum_{j=1}^{m} u_{j} \rho_{j}+\sum_{k=1}^{q} v_{k} \rho_{k} \geqq 0$, then $\lambda^{T}\left(f_{i}(x) / g_{i}(x)\right) \geqq$ $\lambda^{T}\left(f_{i}(y) / g_{i}(y)\right)$.

Proof. By the $(F, \rho)$-convexity of $f_{i}(y) / g_{i}(y), h_{j}(y)$, and $l_{k}(y)$, the sublinearity of $F$, and since $\lambda \in \wedge^{++}, u \in R_{+}^{m}, v \in R_{+}^{q}$, we have that

$$
\begin{aligned}
& \sum_{i=1}^{p} \lambda_{i}\left(\frac{f_{i}(x)}{g_{i}(x)}\right)-\sum_{i=1}^{p} \lambda_{i}\left(\frac{f_{i}(y)}{g_{i}(y)}\right) \\
& \geqq F\left(x, y ; \sum_{i=1}^{p} \lambda_{i} \nabla\left(\frac{f_{i}(y)}{g_{i}(y)}\right)\right)+\sum_{i=1}^{p} \lambda_{i} \rho_{i} d^{2}(x, y), \\
& \sum_{j=1}^{m} u_{j} h_{j}(x)-\sum_{j=1}^{m} u_{j} h_{j}(y) \\
& \geqq F\left(x, y ; \sum_{j=1}^{m} u_{j} \nabla h_{j}(y)\right)+\sum_{j=1}^{m} u_{j} \rho_{j} d^{2}(x, y), \\
& \sum_{k=1}^{q} v_{k} l_{k}(x)-\sum_{k=1}^{q} v_{k} l_{k}(y) \\
& \geqq F\left(x, y ; \sum_{k=1}^{q} v_{k} \nabla l_{k}(y)\right)+\sum_{k=1}^{q} v_{k} \rho_{k} d^{2}(x, y) .
\end{aligned}
$$

By (67) and based on the sublinearity of $F$, we have

$$
\begin{gathered}
\sum_{i=1}^{p} \lambda_{i}\left(\frac{f_{i}(x)}{g_{i}(x)}\right)-\sum_{i=1}^{p} \lambda_{i}\left(\frac{f_{i}(y)}{g_{i}(y)}\right)+\sum_{j=1}^{m} u_{j} h_{j}(x) \\
-\sum_{j=1}^{m} u_{j} h_{j}(y)+\sum_{k=1}^{q} v_{k} l_{k}(x)-\sum_{k=1}^{q} v_{k} l_{k}(y) \\
\geqq F\left(x, y ;\left(\sum_{i=1}^{p} \lambda_{i} \nabla\left(\frac{f_{i}(y)}{g_{i}(y)}\right)\right.\right. \\
\left.\left.+\sum_{j=1}^{m} u_{j} \nabla h_{j}(y)+\sum_{k=1}^{q} v_{k} \nabla l_{k}(y)\right)\right) \\
+\left(\sum_{i=1}^{p} \lambda_{i} \rho_{i}+\sum_{j=1}^{m} u_{j} \rho_{j}+\sum_{k=1}^{q} v_{k} \rho_{k}\right) d^{2}(x, y) .
\end{gathered}
$$

Since $\quad \sum_{i=1}^{p} \lambda_{i} \nabla\left(f_{i}(y) / g_{i}(y)\right)+\sum_{j=1}^{m} u_{j} \nabla h_{j}(y)+$ $\sum_{k=1}^{q} v_{k} \nabla l_{k}(y)=0$, we have

$$
\begin{aligned}
& \sum_{i=1}^{p} \lambda_{i}\left(\frac{f_{i}(x)}{g_{i}(x)}\right)-\sum_{i=1}^{p} \lambda_{i}\left(\frac{f_{i}(y)}{g_{i}(y)}\right) \\
& \geqq \sum_{j=1}^{m} u_{j} h_{j}(y)-\sum_{j=1}^{m} u_{j} h_{j}(x)+\sum_{k=1}^{q} v_{k} l_{k}(y)-\sum_{k=1}^{q} v_{k} l_{k}(x) \\
& \quad+\left(\sum_{i=1}^{p} \lambda_{i} \rho_{i}+\sum_{j=1}^{m} u_{j} \rho_{j}+\sum_{k=1}^{q} v_{k} \rho_{k}\right) d^{2}(x, y) .
\end{aligned}
$$

Since $h_{j}(x) \leqq 0,(j=1,2, \ldots, m), l_{k}(x)=0,(k=1,2, \ldots, q)$, $u^{T} h(y) \geqq 0, v^{T} l(y)=0, \sum_{i=1}^{p} \lambda_{i} \rho_{i}+\sum_{j=1}^{m} u_{j} \rho_{j}+\sum_{k=1}^{q} v_{k} \rho_{k} \geqq 0$, we have $\sum_{i=1}^{p} \lambda_{i}\left(f_{i}(x) / g_{i}(x)\right) \geqq \sum_{i=1}^{p} \lambda_{i}\left(f_{i}(y) / g_{i}(y)\right)$. That is, $\lambda^{T}\left(f_{i}(x) / g_{i}(x)\right) \geqq \lambda^{T}\left(f_{i}(y) / g_{i}(y)\right)$.

\section{Acknowledgments}

This work has been supported by the young and middle-aged leader scientific research foundation of Chengdu University of Information Technology (no. J201218) and the talent introduction foundation of Chengdu University of Information Technology (no. KYTZ201203).

\section{References}

[1] J. von Neumann and O. Morgenstern, Theory of Games and Economic Behavior, Princeton University Press, Princeton, NJ, USA, 1944.

[2] V. Preda, "On efficiency and duality for multiobjective programs," Journal of Mathematical Analysis and Applications, vol. 166, no. 2, pp. 365-377, 1992.

[3] T. R. Gulati and M. A. Islam, "Sufficiency and duality in multiobjective programming involving generalized $F$-convex functions," Journal of Mathematical Analysis and Applications, vol. 183, no. 1, pp. 181-195, 1994. 
[4] J. P. Vial, "Strong and weak convexity of sets and functions," Mathematics of Operations Research, vol. 8, no. 2, pp. 231-259, 1983.

[5] Z. A. Liang, H. X. Huang, and P. M. Pardalos, "Optimality conditions and duality for a class of nonlinear fractional programming problems," Journal of Optimization Theory and Applications, vol. 110, no. 3, pp. 611-619, 2001.

[6] Z. A. Liang, H. X. Huang, and P. M. Pardalos, "Efficiency conditions and duality for a class of multiobjective fractional programming problems," Journal of Global Optimization, vol. 27, no. 4, pp. 447-471, 2003.

[7] T. Weir and B. Mond, "Generalised convexity and duality in multiple objective programming," Bulletin of the Australian Mathematical Society, vol. 39, no. 2, pp. 287-299, 1989.

[8] T. Weir, "A duality theorem for a multiple objective fractional optimization problem," Bulletin of the Australian Mathematical Society, vol. 34, no. 3, pp. 415-425, 1986.

[9] V. Jeyakumar and B. Mond, "On generalised convex mathematical programming," Australian Mathematical Society B, vol. 34, no. 1, pp. 43-53, 1992.

[10] R. R. Egudo, "Efficiency and generalized convex duality for multiobjective programs," Journal of Mathematical Analysis and Applications, vol. 138, no. 1, pp. 84-94, 1989.

[11] A. Cambini and L. Martein, Generalized Convexity and Optimization, Springer-Verlag, Berlin, Germany, 2008.

[12] L. Cuoyun and D. Jiali, Methods and Theories of Multi-Objective Optimization, Jilin Education Press, Changchun, China, 1992.

[13] W. Zezheng and Z. Fenghua, "Optimality and duality for a class of nonlinear fractional programming problems," Journal of Sichuan Normal University, vol. 30, no. 5, pp. 594-597, 2007.

[14] O. L. Mangasarian, Nonlinear Programming, McGraw-Hill, New York, NY, USA, 1969. 


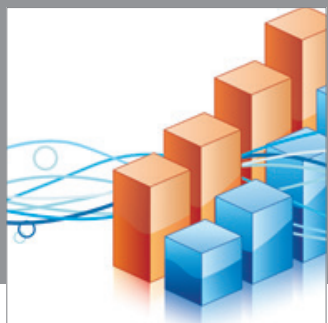

Advances in

Operations Research

mansans

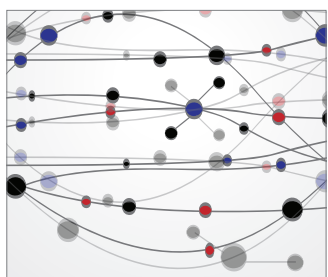

The Scientific World Journal
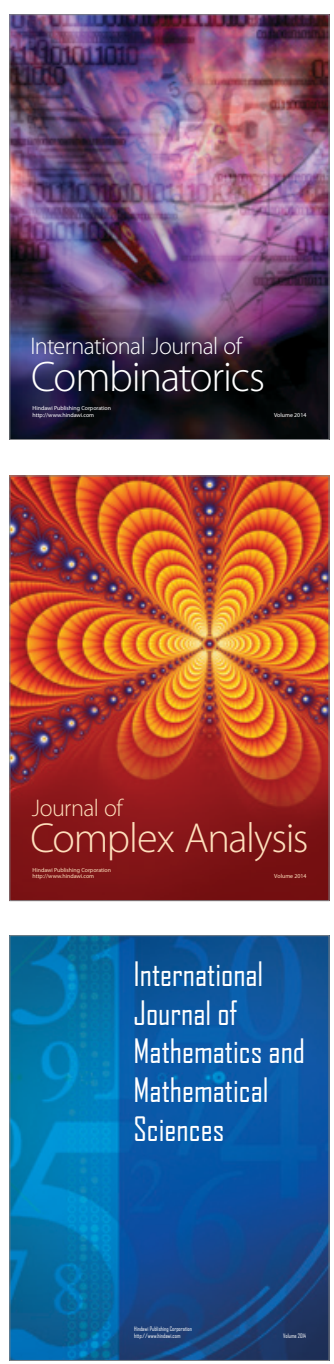
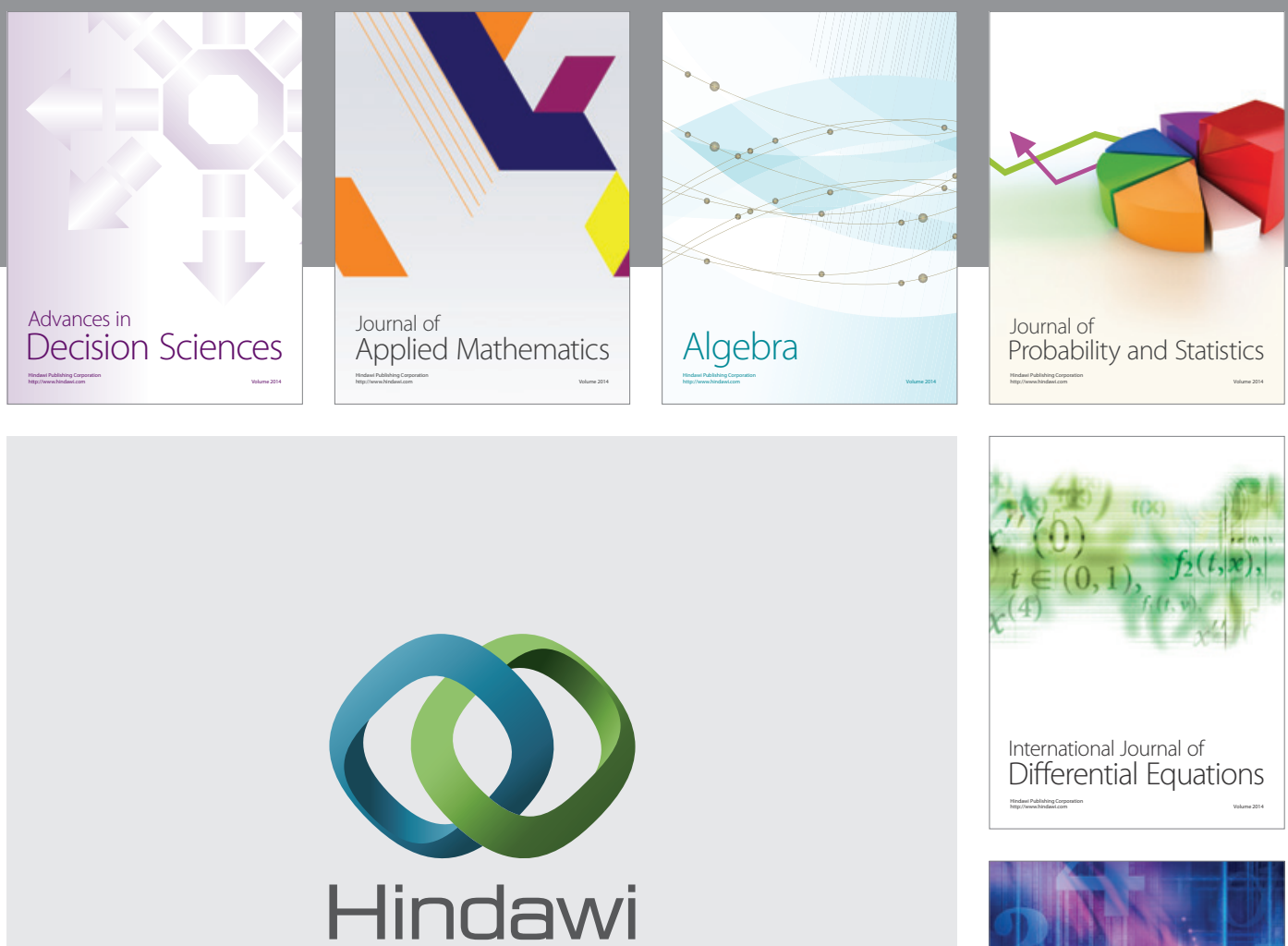

Submit your manuscripts at http://www.hindawi.com
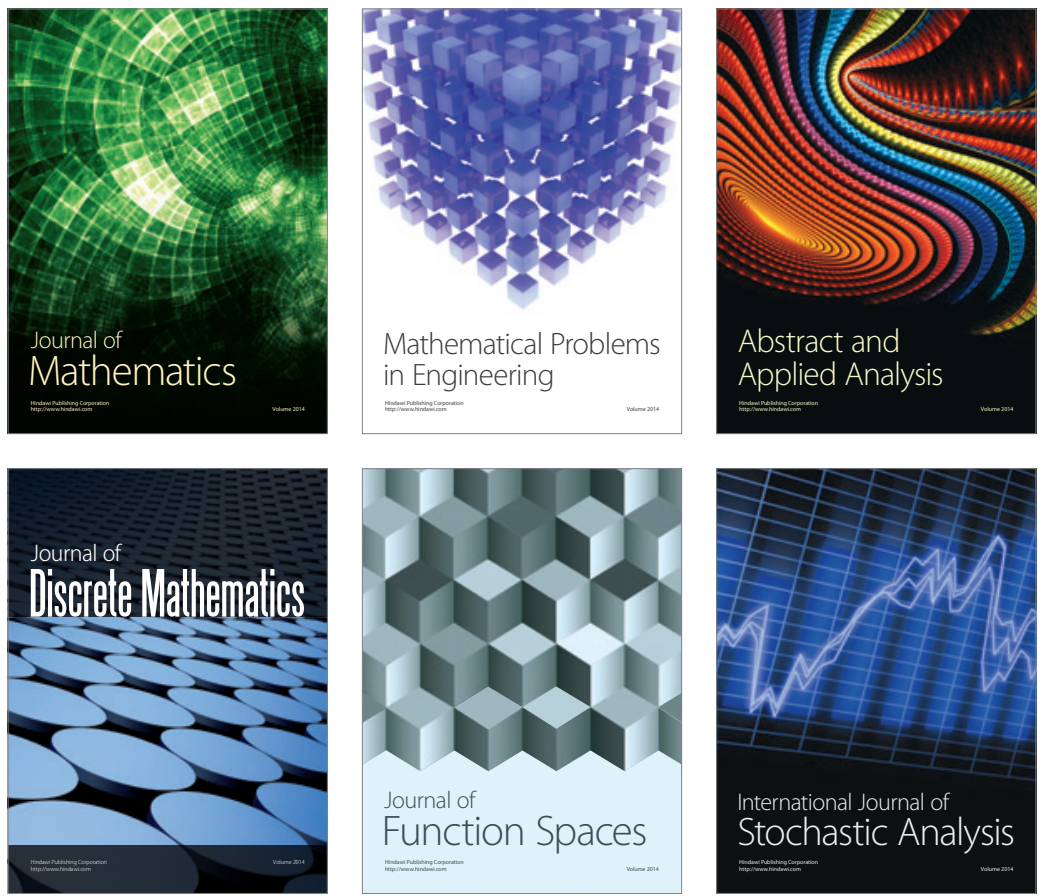

Journal of

Function Spaces

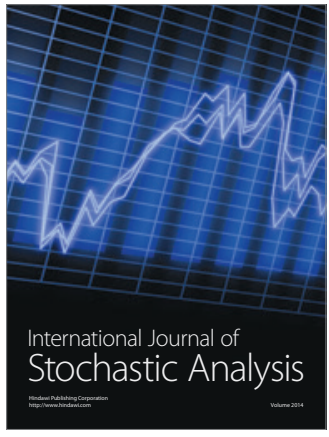

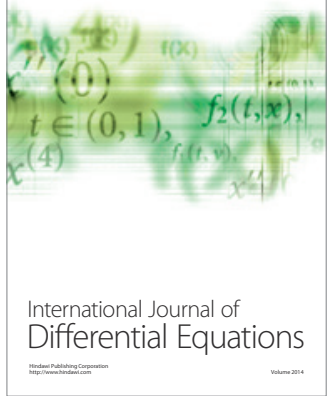
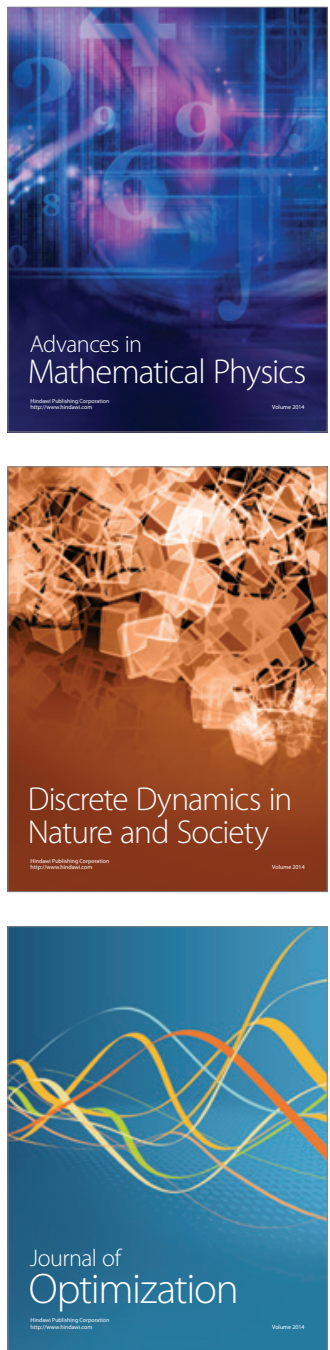